\title{
Jet formation in bubbles bursting at a free surface
}

\author{
Laurent Duchemin, Stéphane Popinet, Christophe Josserand \\ and Stéphane Zaleski
}

\begin{abstract}
We study numerically bubbles bursting at a free surface and the subsequent jet formation. The Navier-Stokes equations with a free surface and surface tension are solved using a marker-chain approach. Differentiation and boundary conditions near the free surface are satisfied using least-squares methods. Initial conditions involve a bubble connected to the outside atmosphere by a preexisting opening in a thin liquid layer. The evolution of the bubble is studied as a function of bubble radius. A jet forms with or without the formation of a tiny air bubble at the base of the jet. The radius of the droplet formed at the tip of the jet is found to be about one tenth of the initial bubble radius. A series of critical radiuses exist, for which a transition from a dynamics with or without bubbles exist. For some parameter values, the jet formation is close to a singular flow, with a conical cavity shape and a large curvature or cusp at the bottom. This is compared to similar singularities investigated in other contexts such as Faraday waves.
\end{abstract}

\section{Introduction}

Bubbles bursting at the water surface are a familiar everyday occurrence. They also take part in important processes of transport and exchange across liquid/gas interfaces, caused by the ejection of jets and various kinds of small droplets. These are involved in the transfer of heat, mass and various contaminants between the oceans and the atmosphere [1]. Indeed, breaking waves cause the formation of a large number of bubbles beneath the water 
level. The efficiency of the resulting mass transfer, including the transfer of $\mathrm{CO}_{2}$ depends on the initial properties of the ejected droplets (size, initial velocity).

The phenomena producing aerosols during the bursting of a bubble are of two kinds: the first is the rupture of the film separating the bubble from the atmosphere. This film atomization can produce several hundred droplets of around a micrometer in diameter which probably represent a large fraction of the transfers [1]. Since the scales involved during this rupture are of the order of $100 \mathrm{~nm}$, a physical description is outside the scope of continuum fluid mechanics. Indeed, long-range molecular forces such as Van der Waals forces or electrostatic repulsion must be taken into account [2].

The small cavity remaining after the film rupture collapses under the effect of both surface-tension and buoyancy. This collapse gives birth to a narrow vertical jet which eventually breaks into one or several droplets (see Fig. 1). This phenomenon constitutes the second aerosol production process and is the principal topic of this paper.

These aerosols are of a different kind: they are ejected vertically — which is not the case for film aerosols - and their diameter is about one tenth of the size of the initial cavity, i.e. about $100 \mu \mathrm{m}$ for a typical bubble radius of one millimeter. Depending on their mass and initial velocity, the droplets will either fall back into water or evaporate.

The topic of this paper is the investigation of the bubble evolution after the initial film rupturing, including the jet formation. A numerical method solving the Navier-Stokes equations and describing the free surface with high precision is used. Previous numerical studies of these phenomena have been made postulating mostly inviscid fluids; however, a modified boundary element method taking into account small viscous effects was also used [2, 3]. A Navier-Stokes simulation was shown in [4], with a VOF-type method in a regime where the bubble is very deformed.

In most previous studies the effect of film atomization on jet birth was assumed to be negligible. Few comparisons were made with experimental data. Some experimental studies were also conducted to measure quantities such as jet velocity $[5,6,7]$, size of the first ejected droplet, height at which the droplet detaches from the jet, or height reached by the droplet. These experiments are fairly difficult to conduct, because of surface contamination which modifies significantly the free-surface boundary condition and the surface tension coefficient.

As our numerical results will demonstrate, the jet formation is in many 


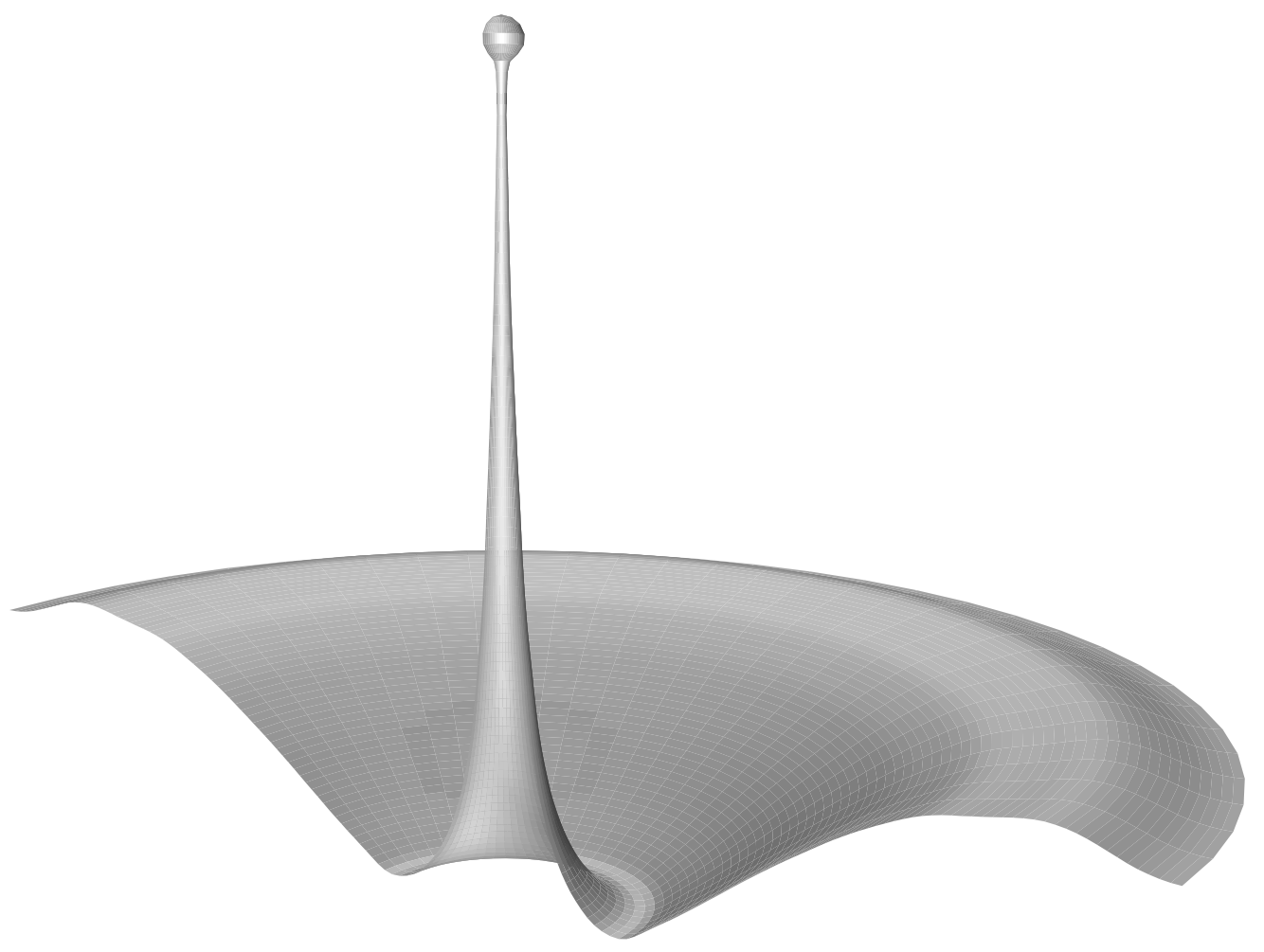

Figure 1: Jet produced by the collapse of a spherical cavity. The end droplet will eventually detach due to the Savart-Plateau-Rayleigh instability. 
cases singular and self-similar. Singular jets forming at a free surface have already been observed and studied in different contexts. Indeed, in the bubblebursting problem as well as in several other free-surface flows, one observes the formation of a conical cavity, with a very high curvature or cusp at its base. In some cases a small bubble is trapped at the bottom of the cavity. A thin narrow jet subsequently forms in a self-similar manner. This phenomenon was observed experimentally in Faraday waves by Longuet-Higgins [8] and Lathrop [9], in the development of the jet inside a bubble containing a sink flow in the numerical study of [10]. For bursting bubbles the conical cavity may be seen in the experiments and simulations but the singular character of the jet formation has not been investigated to our knowledge. The phenomenon may also be seen in the cavity formed by falling raindrops $[11,12,13,14]$.

The evolution of the conical cavity has been studied by Longuet-Higgins [12] as a special case of a family of hyperbolic surfaces : conical surfaces were shown to be a special case of the hyperbolic surfaces of Ref. [8]. These conical surfaces are preserved by the velocity potential

$$
\Phi=A(t) r^{2} P_{2}(\cos \theta)
$$

where $r$ is the spherical radial distance and $\theta$ the polar angle measured from the north pole, which yields the velocity field

$$
\nabla \phi=A(t)(-x,-y, 2 z)
$$

where $A(t)$ is an arbitrary function. Indeed any conical free surface in this flow remains conical. For positive $A$ the cavity opens in time as in the experiment.

Of course the actual flow is not exactly conical. The bottom of the cone is rounded, and oscillates in shape as capillary waves converge towards the bottom of the conical surface. At some instant in time the bottom may develop a cusp, followed by jet formation. This process is obviously singular at least for some values of the parameters, but there is no agreement among the above cited publications on the exact nature of the singularity.

Indeed one may inquire into the specific scaling form of the singularity. The Euler equations without surface tension and gravity will in principle admit self-similar solutions of the form

$$
\Phi(\mathbf{x}, t)=\left|t-t_{0}\right|^{m} \varphi\left(\mathbf{x}\left|t-t_{0}\right|^{-n}\right)
$$


where $\varphi$ is an arbitrary scaling function and $t_{0}$ is the singularity time. The solution may be valid before and/or after the singularity time. The exponents should satisfy $m=2 n-1$. Indeed with this condition all the terms in the Bernoulli equation balance. However when surface tension is added, the only way to form a self-similar solution that balances inertia and surface tension is by selecting $n=2 / 3$. This is because the only length scale that can be built is $r_{c}=\left(\sigma t^{2} / \rho\right)^{1 / 3}$ where $\sigma$ is the surface tension and $\rho$ the density. Then the similarity variable is $\eta=\mathbf{x} / r_{c}$ and the flow velocity diverges like $t^{-1 / 3}$ near the singularity for a fixed value of the similarity variable $\eta$. This idea is at the basis of 2/3 exponents found for instance by Miksis and Keller [15]. This type of scaling, was applied by Zeff et al. [9] to observations of jet formation in Faraday waves. The leading order term for the velocity potential is of the form

$$
\Phi=C\left|t-t_{0}\right|^{1 / 3} r^{1 / 2} P_{1 / 2}(\cos \theta) .
$$

However, a series of alternate theories for singular free-surface flows and in particular the conical cavity and jet formation was proposed by LonguetHiggins. He has shown that the type of flow described by Eq. (2) had a divergent velocity with $A(t) \sim\left|t-t_{0}\right|^{-1 / 3}$ thus a $t^{-1 / 3}$ divergence for a fixed value of the real (unscaled) distance $r$ [12]. In this solution the scaling is not fixed by a balance with surface tension. Instead, surface tension is added as a perturbation to the conical solution, in the form of a sink flow [12]. The Longuet-Higgins solution yields an angle for the conical cavity of $2 \theta=109^{\circ} 5$, in good agreement with the numerical observations of [11]. Another self-similar solution for jet formation was found numerically by [10] obtaining yet another scaling, for the case of jet formation inside a bubble. The potential is then approximated by

$$
\Phi=A(t) r^{1 / 4} P_{1 / 4}(\cos \theta) .
$$

This article is organized as follows. We first describe the general context of this study, the non-dimensional numbers controlling the problem and the scaling laws deduced from dimensional analysis. We then briefly introduce the numerical method we use and its main advantages. A first comparison with experimental profiles is presented. Finally, a detailed parametric study is conducted using a simple initial shape for the cavity and neglecting gravity. We measure the volume of the first ejected droplet, the velocity of the jet and the maximum pressure encountered on the axis of symmetry and discuss the results. In some circumstances, a tiny bubble is formed at the base of 
the jet. The self-similar flow occurring when the conical cavity and the cusp form is investigated.

\section{Initial conditions and expected scaling laws}

Given the small size of the bubbles we are interested in (diameter is around one millimeter), some assumptions can be made regarding the parameters governing jet birth. The first idea is to suppose that the cavity is motionless at the initial time. Experiments have shown that, even in the absence of surfactants, the bubble can stay at the free surface in a quasistatic equilibrium for a few seconds [16]. The bubble is then separated from the atmosphere by a thin liquid film, the cavity being subject to surface tension and buoyancy forces. A model for this static configuration is a more or less deformed bubble adjacent over part of its surface to a film of negligible thickness. This configuration may be computed, or obtained from the experimental data as in the case reported in [7].

When the film reaches a critical thickness (about $100 \mathrm{~nm}$ ) after draining slowly, it breaks more or less rapidly (depending on the presence of surface contaminants). It is then possible to run simulations by taking the current, static configuration and removing the thin film. While we do this in one case, the drawback is that a sharp corner exists at the rim of the neck or juncture between the film and the bulk liquid. The small length scales involved may create numerical convergence problems. Moreover, as we show below, small length scales are generated independently of initial conditions by the steepening of capillary waves and jet formation. Keeping the small length scales in the initial conditions makes it more difficult to observe the intrinsically generated small scales. We thus decided to drastically smooth the rim of the neck. In most calculations, the initial shape was defined as follows. A spherical cavity is separated from the atmosphere by a circular hole, the border of the hole being a circular rim (see Fig.2).

The collapse behavior depends only on four physical parameters: the kinematic viscosity $\nu, \sigma, \rho$ and the acceleration due to gravity $g$. Out of the four physical parameters only two length scales can be defined, the capillary length $R_{c}=(\sigma / \rho g)^{1 / 2}$ and the the viscous-capillary length $R_{\nu}=\rho \nu^{2} / \sigma$. In pure water $R_{c}=2.7 \mathrm{~mm}$ and $R_{\nu}=0.014 \mu \mathrm{m}$ respectively. If the radius of the bubble $R \ll R_{c}$, capillary effects are predominant compared with the gravity effects; if $R \gg R_{\nu}$, viscous effects are expected to be negligible compared 
to the capillary ones. For $R_{\nu} \ll R \ll R_{c}$ the phenomenon is dominated by surface tension and inertia.
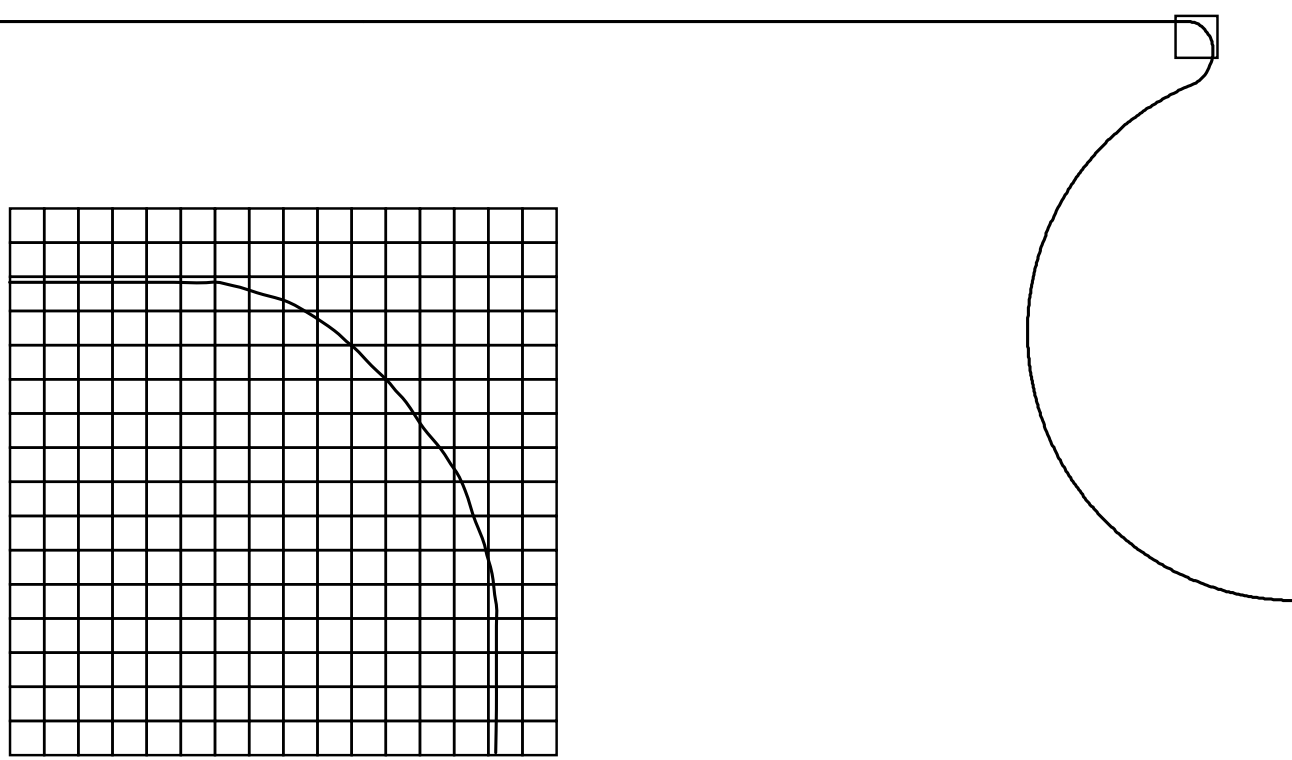

Figure 2: The initial configuration in the "large rim" case. The grid is a $512^{2}$ Cartesian grid.

We also decided to neglect the effect of gravity which is a correct approximation for $R \ll R_{c}$. Therefore, only the Ohnesorge number $Z=\rho \nu^{2} / \sigma R$ governs the phenomenon and dimensional analysis gives velocity in the form

$$
V \sqrt{\frac{\rho R}{\sigma}}=F\left(\frac{\rho \nu^{2}}{\sigma R}\right)
$$


where $F$ is an unknown function.

Whenever $R_{\nu} \ll R \ll R_{c}$, we also expect that viscosity plays no role. The only way to eliminate viscosity is to suppose that the function $F$ has a finite, non-zero limit $C$ when $\nu$ goes to zero. The non-dimensional velocity of the jet then behaves like

$$
\frac{V}{V_{\nu}} \simeq C\left(\frac{R}{R_{\nu}}\right)^{-1 / 2}
$$

where $V_{\nu}=\sigma / \rho \nu$.

Similar arguments lead to a scaling law for the non-dimensional pressure of the form

$$
\frac{P}{P_{\nu}} \sim C^{\prime}\left(\frac{R}{R_{\nu}}\right)^{-1}
$$

where $P_{\nu}=\sigma^{2} / \rho \nu^{2}$.

\section{$3 \quad$ Numerical method}

The choice of the numerical method is conditioned by the terms we need to solve accurately. In our problem, the first term of interest is surface tension: being the main driving force in the parameter range we consider, it is important to model it correctly. Given the large density ratio between water and air we can moreover assume that the influence of the gas phase is negligible.

According to these two assumptions, we used a numerical method which solves the full axisymmetric Navier-Stokes equations in a fluid bounded by a free surface while allowing an accurate description of the interfacial terms such as surface tension. This method has been documented elsewhere $[17,18]$ and has been shown to produce accurate qualitative and quantitative results when compared with both theoretical and experimental data.

In short, a regular Cartesian fixed grid is used. Massless particles (markers) advected by the flow define the position of the interface. Linked by cubic splines, they describe accurately the geometry of the free surface. For cells which are not cut by the free surface, a classical finite-volume scheme is applied. For the cells in the vicinity of the interface, finite differences cannot be computed since velocities are not defined in the "gas" phase. Therefore, an extrapolation of the velocity field near the free surface on the other side is necessary. This extrapolation must take into account the boundary 
conditions on the free surface (in particularly the nullity of the tangential stress). This is done by using a least-mean-square procedure constrained by the condition of vanishing tangential stress. Comparisons with theoretical results show that this approach gives an accurate description of the viscous dissipative terms associated with the boundary conditions.

The pressure on the boundary is obtained as follows. The local curvature is estimated from the spline reconstruction. The local normal viscous stress is estimated from the above least-squares procedure. Then the pressure is obtained from the normal-stress boundary condition. The pressure on the boundary serves as a boundary condition for the Poisson equation for the pressure. This equation is in turn solved using a multigrid algorithm.

Most computations have been made on a $512^{2}$ grid, except the comparison with the experimental profiles from MacIntyre, which has been made on a $1024^{2}$ grid and some selected computations which were refined to $1024^{2}$ grids.

\section{Comparison of the numerical results with experimental profiles}

We have first initialized the calculation with a realistic shape, and taken into account all the physical parameters, i.e. capillarity, viscosity and gravity. The goal was to compare the results with a series of shapes published by MacIntyre [7]. The initial shape of the free surface has been obtained from the first image given by MacIntyre, just after the film rupture.

Fig. 3 illustrates the experimental and the computational results. The numerical parameters are: $\nu=10^{-6} \mathrm{~m}^{2} / \mathrm{s}, \sigma=0.072 \mathrm{~kg} / \mathrm{s}^{2}, \rho=1000 \mathrm{~kg} / \mathrm{m}^{3}$ and the volume of the bubble is the same as the one given by MacIntyre: $2.57 \mu \mathrm{l}$. The computational time is about one day on the $1024^{2}$ grid. The overall agreement is very satisfactory. In particular capillary waves are well described, in contrast to the earlier published results using boundary integral methods $[3,2]$. We believe that this lack of capillary waves is due to the strong smoothing needed to avoid numerical instabilities in boundary integral techniques (and probably also to an insufficient spatial resolution, which is also limited by numerical stability). In our method, real, molecular viscosity is present and the fine grid we use allows in principle to solve the small spatial scales of the capillary waves.

The time interval between images is the same as the one given by Mac- 

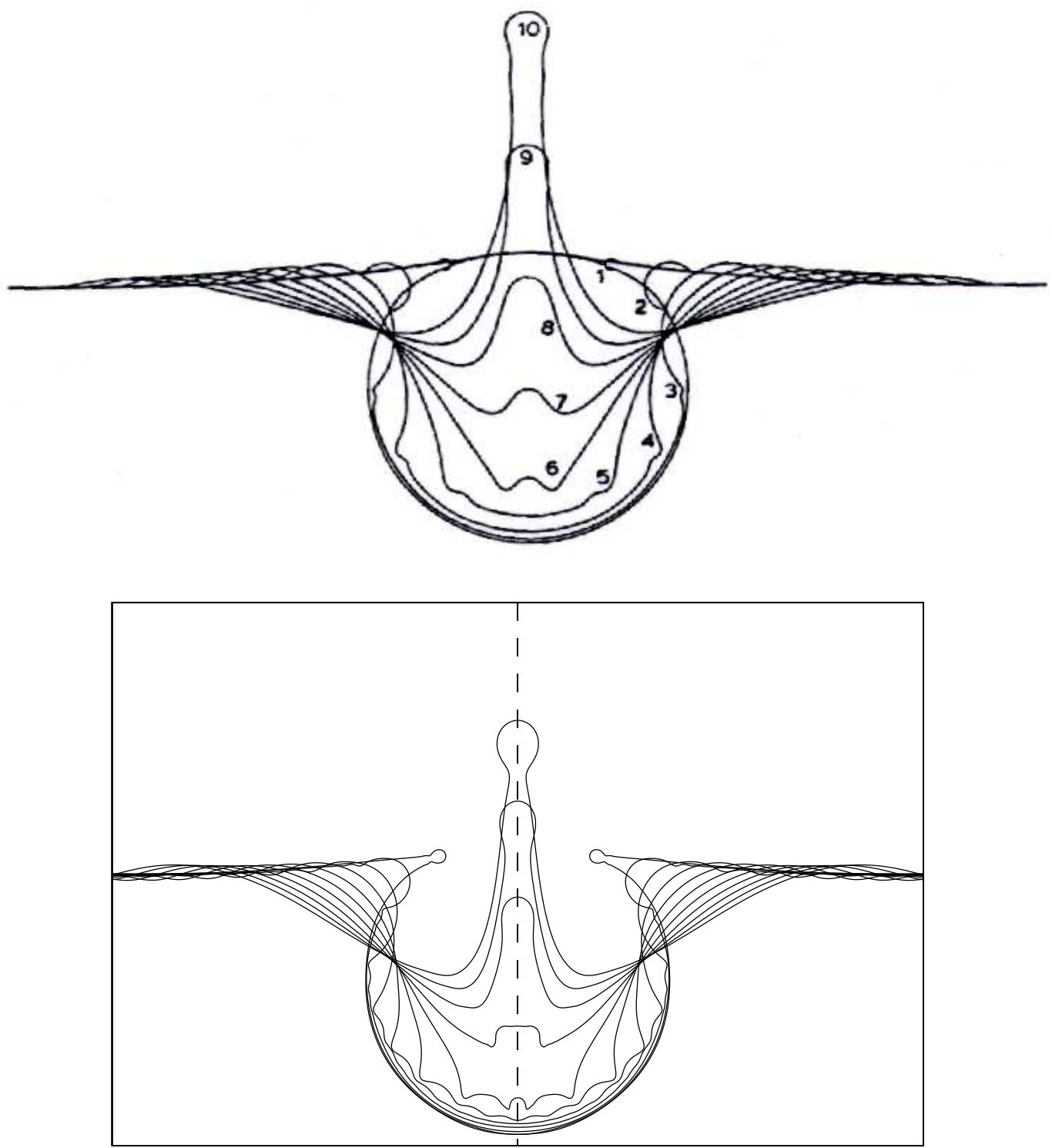

Figure 3: Time sequence of the jet formation in a $2.57 \mu l$ bubble bursting at a free surface. Top: experimental [7] and bottom: computational results. Profiles are $1 / 6000$ s. apart. 
Intyre, i.e. $1 / 6000 s$. A difference in time between profiles can be seen, even if the shape is very similar. A possible explanation is the presence of surface contaminants in the MacIntyre experiment. These contaminants could change the surface tension, even modify its value locally, therefore changing the behavior of the free surface through generation of Marangoni currents. They could also make the interface partially or entirely rigid, changing the free-surface boundary condition.

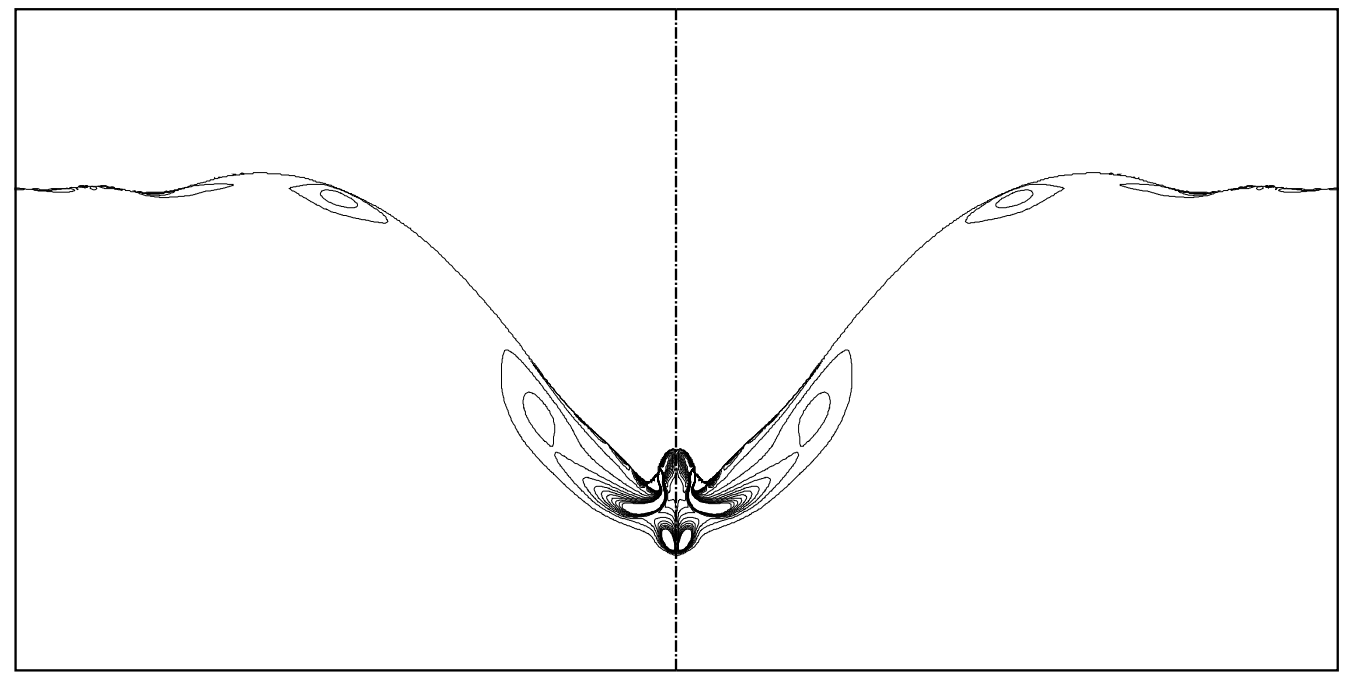

Figure 4: Vorticity isolines during the collapse of the bubble for the same conditions as in Fig. 3. 


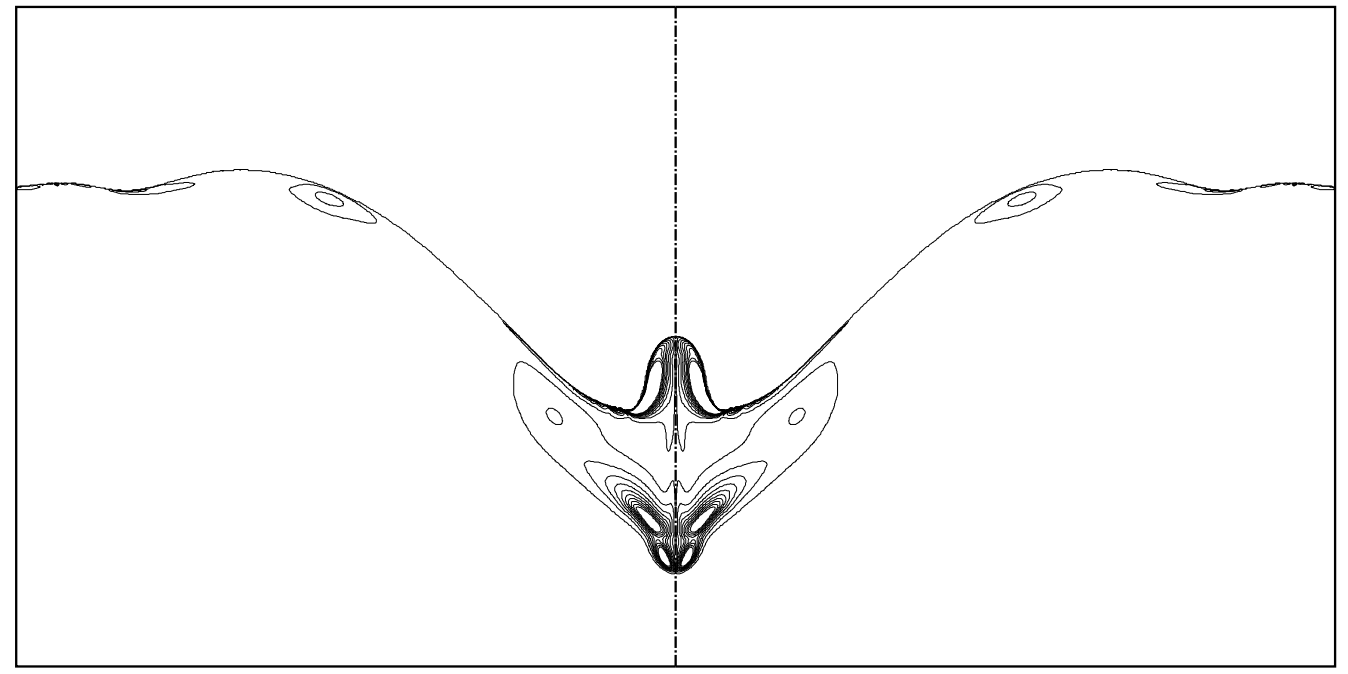

Figure 5: Same as previous figure.

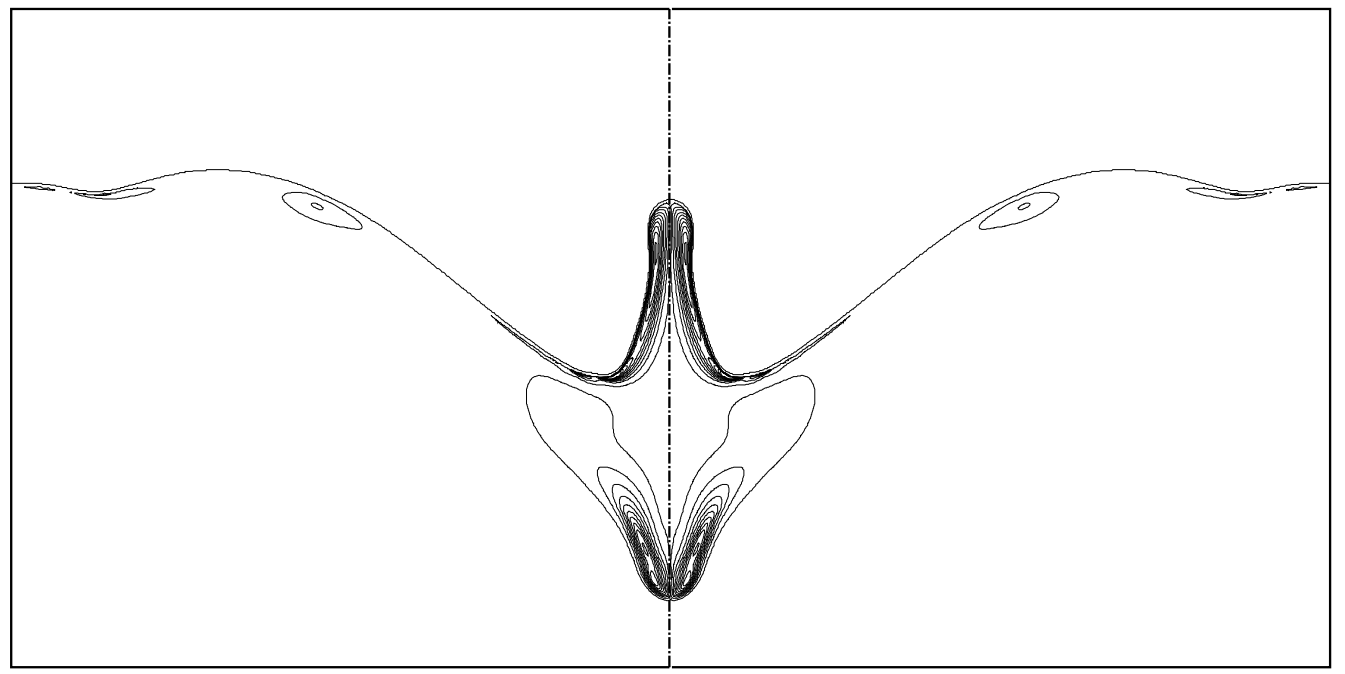

Figure 6: Same as previous figure. 


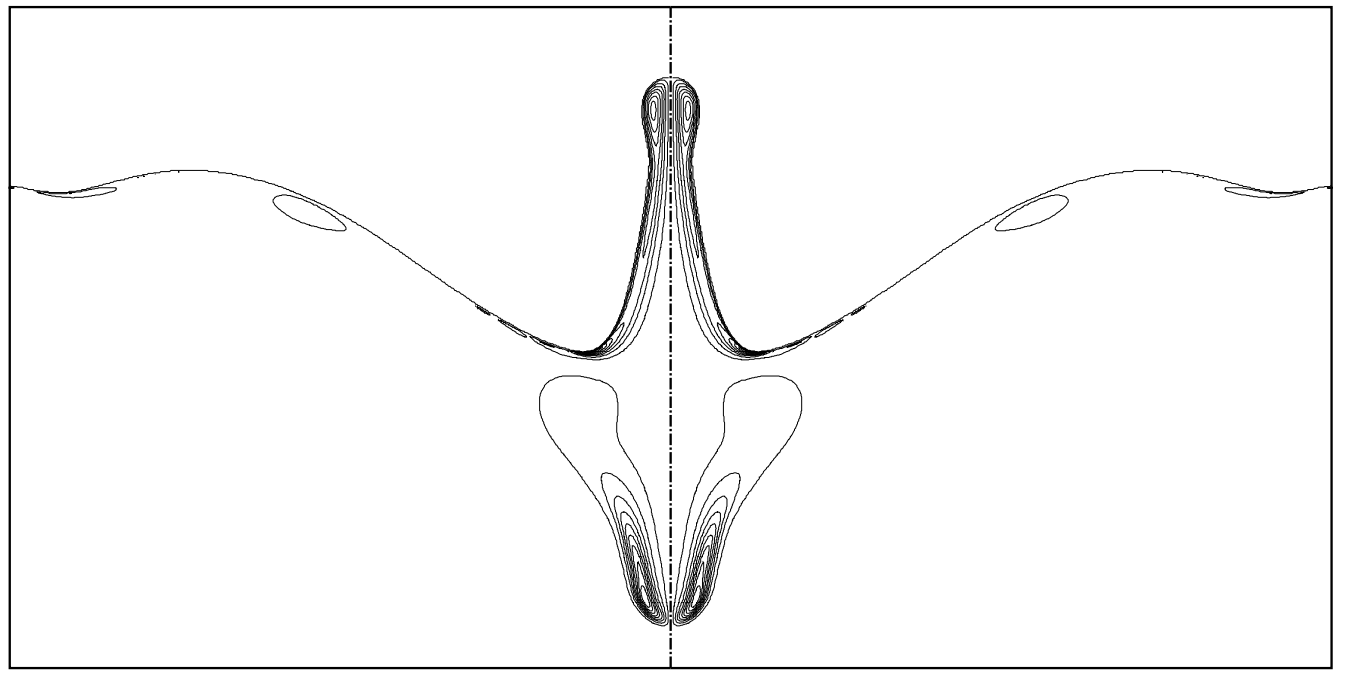

Figure 7: Same as previous figure . 
Solving the full Navier-Stokes equations, we have access to vorticity which can, as we will see later, have an important effect even on very small structures in a low viscosity fluid such as water. Fig. 4 shows the vorticity isolines during the collapse of the cavity. The vorticity is confined to a thin boundary layer before the jet birth. Later on however, a vorticity cone is entrained below the jet and the shear stress there is comparable to that in the narrow jet. This detachment of vorticity illustrates the formation of a downward jet, already observed by Boulton-Stone and Blake with their modified boundary integral method $[3,2]$.

\section{Results of the parametric study}

A set of computations have been made for radii between $10^{-6} \mathrm{~m}$ and $10^{-2} \mathrm{~m}$ $\left(10^{2}<R / R_{\nu}<10^{6}\right.$ ) with the initial shape described above.

The evolution of the profiles is very similar to that shown on Fig. 3. A conical cavity forms with a train of capillary waves converging to the axis. The number of capillary waves depends strongly on the Ohnesorge number: the higher this number, the higher the number of capillary waves converging to the base of the cavity. Fig. 8 shows a large-radius case with a large number of waves (see also Fig. 11). In some cases, especially near $R / R_{\nu}=10^{3}$ the jet became very thin (Fig. 9) and the local radius of curvature smaller than the grid size. The calculation then becomes inaccurate and has to be stopped.

For some parameter values we observe an tendency to trap a bubble on the axis of symmetry just before the formation of the jet. We have searched systematically for bubble entrapment. There are two competing changes of shape: the jet formation is heralded by a change of curvature at the base of the cavity, while the bubble pinching is preceded by the formation of an overhang in the interface, i.e. the height $h(r)$ of the interface becomes multivalued. Thus our criterion for incipient bubble formation is as follows: (a) The height $h(r)$ becomes steep, then multi-valued, and (b) the curvature at the base remains positive. This is only an indication that a bubble will be trapped before the jet forms as shown on Fig. 10, but we need such a crude criterion because the bubbles are very small for the kinds of grids we have. We found a first bubble entrapment region for $576<R / R_{\nu}<2016$, the second one between $57600<R / R_{\nu}<288000$. Other such regions at higher values of $R / R_{\nu}$ are likely, but difficult to observe numerically. One indication is the existence of large trains of capillary waves at large $R / R_{\nu}$ as shown on 


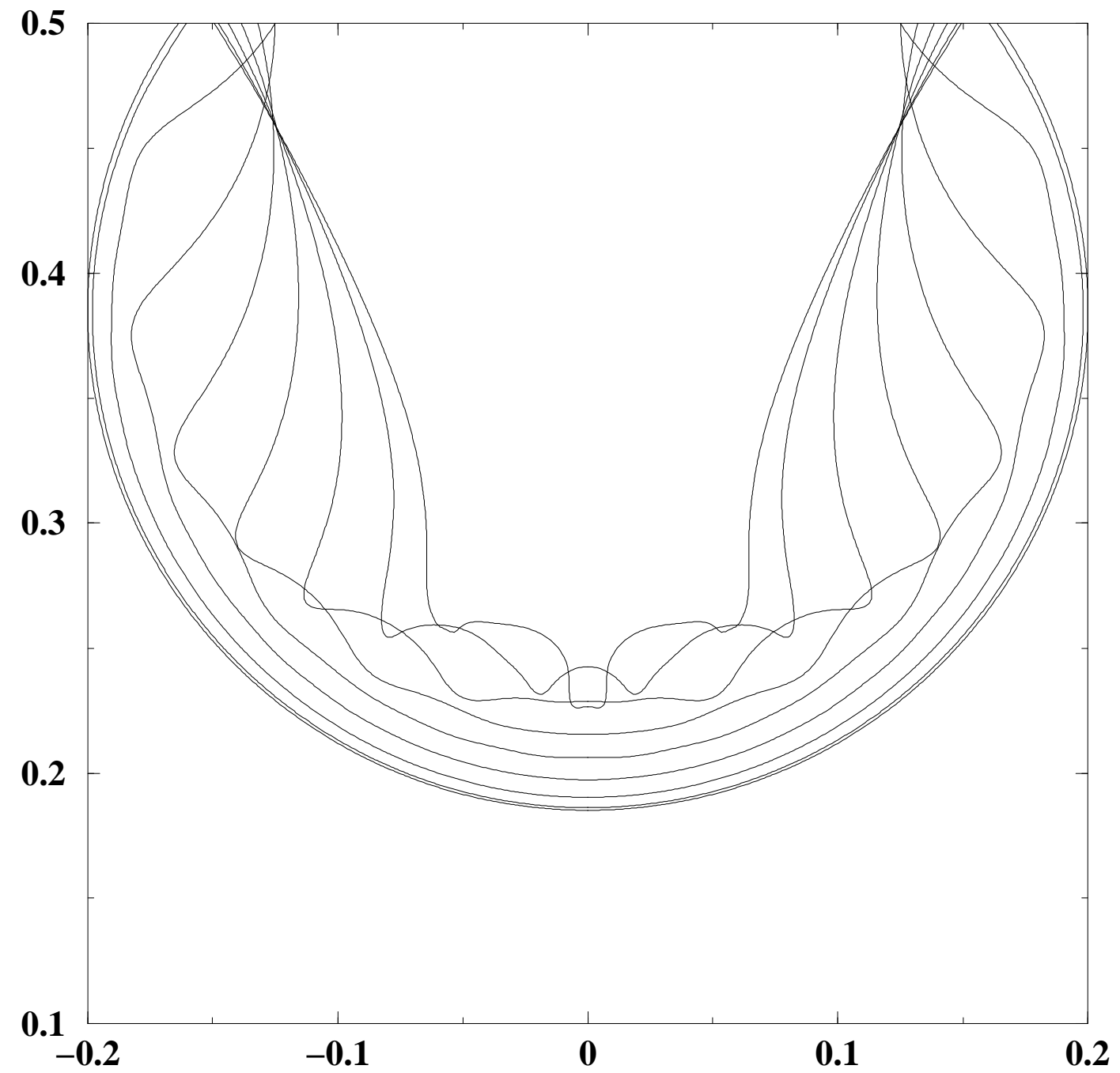

Figure 8: Capillary waves for $R / R_{\nu}=14400$. As $R / R_{\nu}$ 


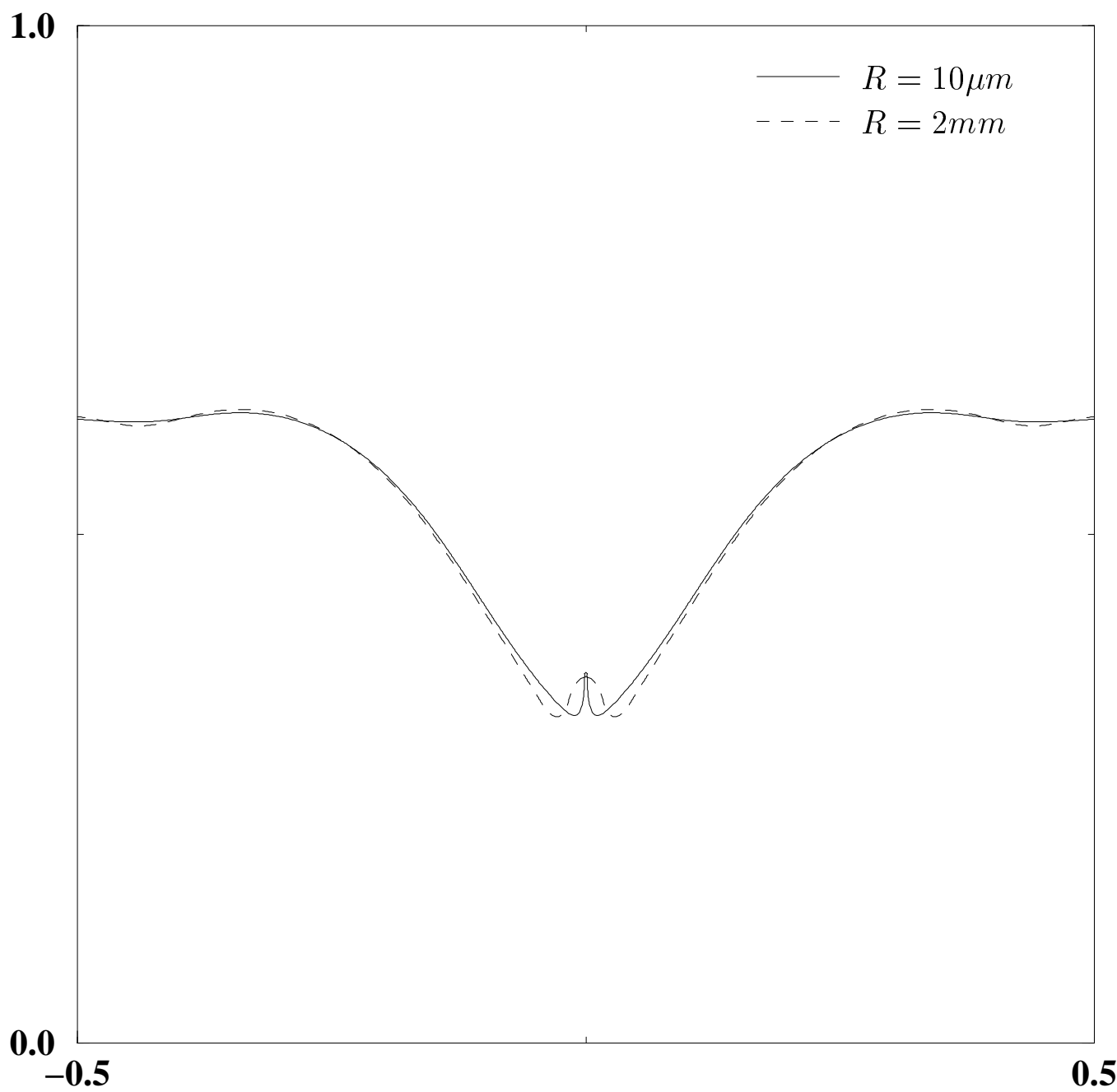

Figure 9: The initial phase of jet formation as seen in two simulations. For the large bubble (dashed line, $R / R_{\nu}=14400$ ) the jet is relatively wide and well resolved numerically. For smaller bubbles (solid line, $R / R_{\nu}=720$ ) the jet may become extremely thin. 
Fig. 11.

The topology of the interface changes when a bubble is trapped. This pinching is a singular event akin to the pinching of a gas cylinder by the Savart-Plateau-Rayleigh instability. We shall call it a pinching singularity to distinguish it from other free surface singularities. To pursue the calculation numerically beyond a pinching singularity, one should in principle perform surgery on the marker chain and continue the simulation. This is however difficult because the problem slightly changes in nature: the pressure inside the small trapped bubble cannot be set to atmospheric pressure but should in principle depend on the bubble volume through some equation of state. This changes markedly the nature of the calculation. Moreover the trapped bubbles are extremely small and very difficult to resolve without mesh adaptation. Thus in most cases we continued the simulation without marker surgery. When the trapped bubble is very small, the marker chain reorganizes itself spontaneously and the calculation proceeds. In some cases, as in the rightmost bubble entrapment region, it seems that the effect on the dynamics is small. In other cases, as in the leftmost entrapment region, the calculation has to be stopped or provides unreliable results which were removed from the quantitative analyses below.

We have redone all the calculations for a different initial condition. The overall configuration is the same as on Fig. 2 but the rim thickness is halved. All the above qualitative results are identical. In particular, we do not observe any steepening of the capillary waves or thinner jets as we reduce the rim size. This is a clear indication that the small length scales we observe form spontaneously, independently of initial conditions.

\subsection{Jet velocity}

A first quantity of interest is the velocity of the jet, or the ejection speed of the first drop. Fig. 12 shows the non-dimensional velocity of the jet, measured when the top of the jet reaches the mean water level. Circle symbols correspond to the larger rim thickness as on Fig. 2 while square symbols correspond to thinner rims. Apart from a vertical shift, the measured velocities are very similar. This shift may in part be explained by the fact that we measure the jet velocity at the mean water level for both cases, which is at a different distance from the base of the two cavities.

For a large range of radii (between $2 \times 10^{4}$ and $10^{6}$ times the viscouscapillary length $R_{\nu}$ ), the numerical results are in good agreement with the 


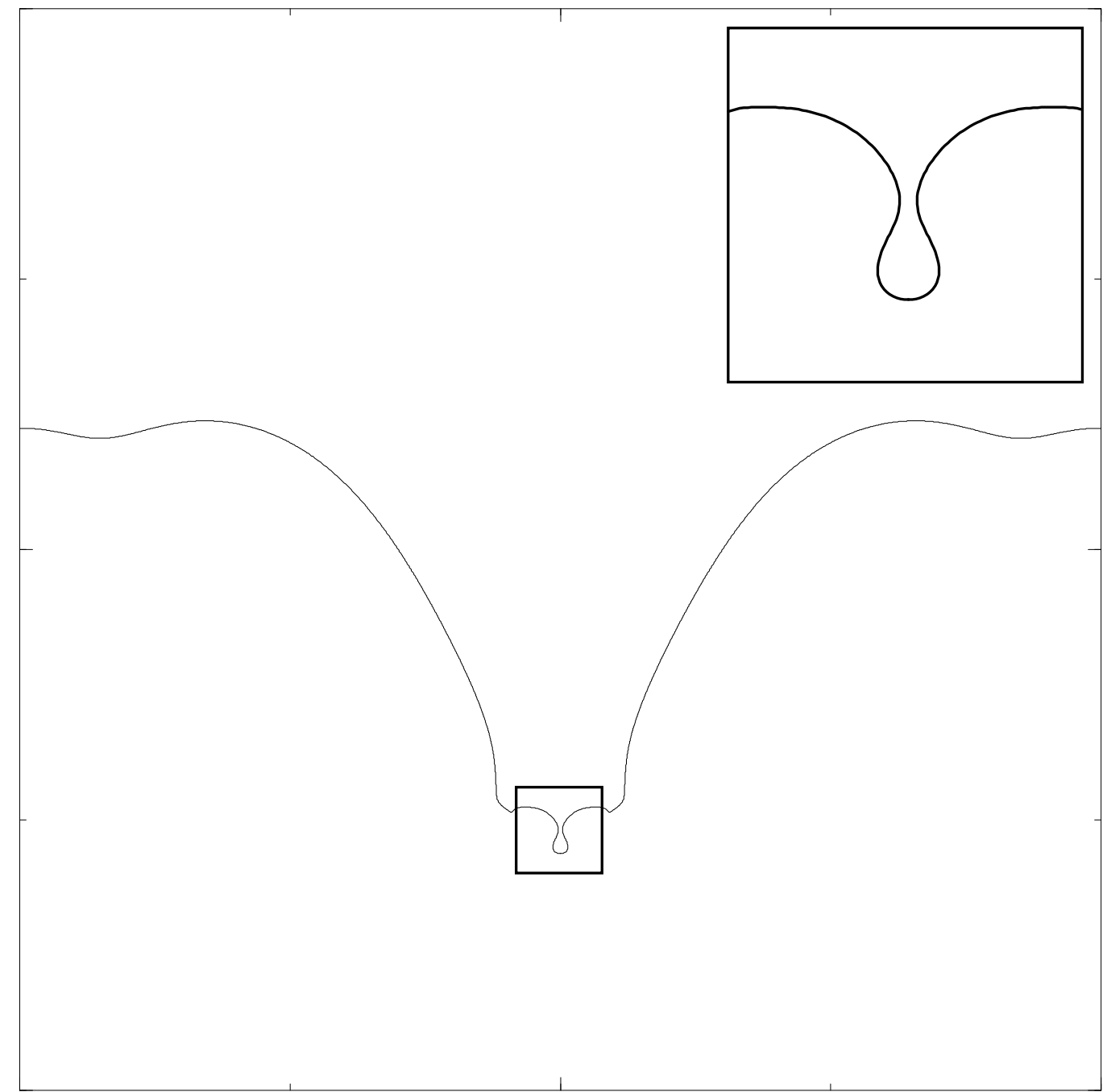

Figure 10: Beginning of the entrapment of a bubble by the collapsing cavity, for $R / R_{\nu}=10^{5}$ (a $1.4 \mathrm{~mm}$ bubble). 


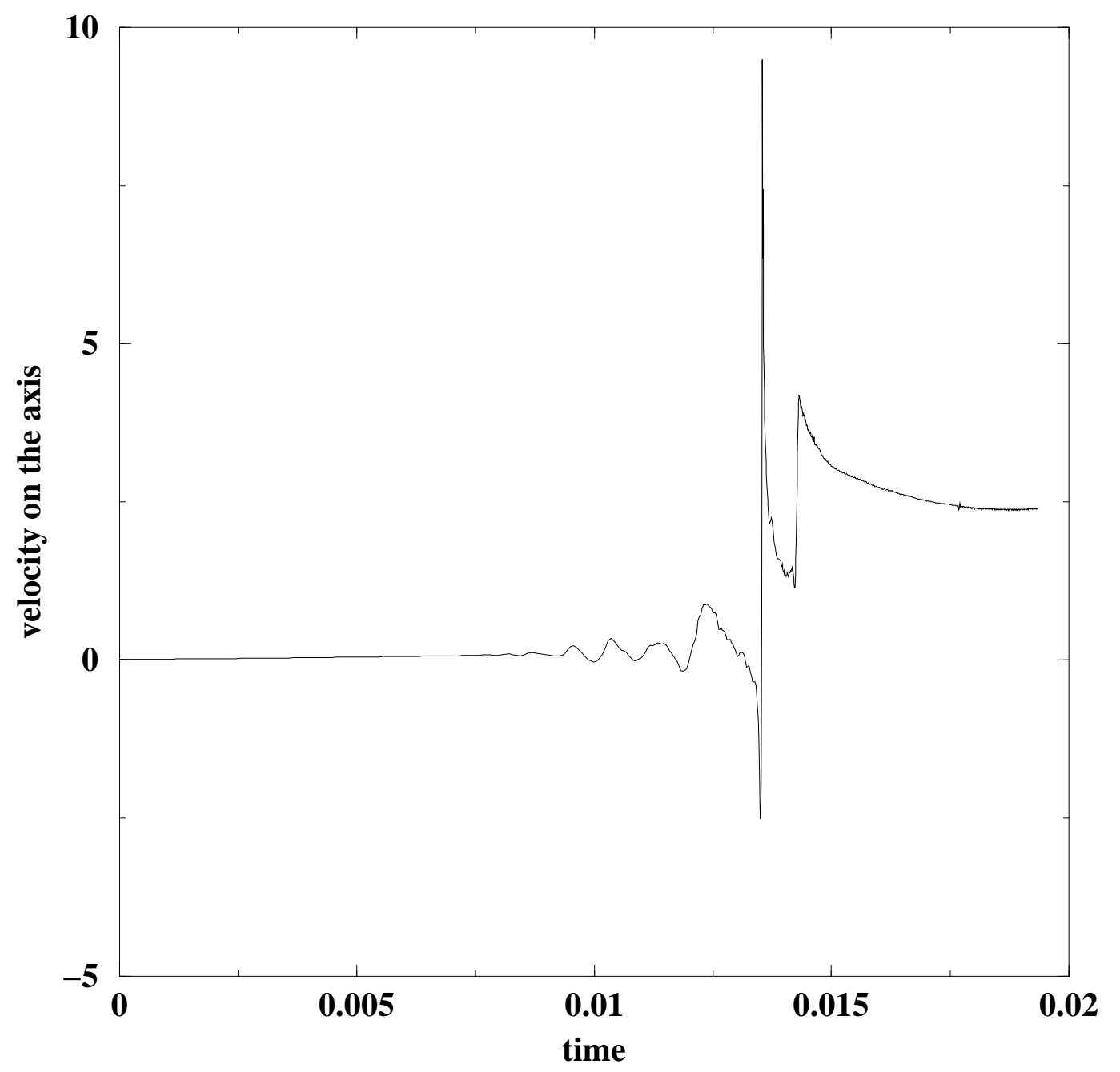

Figure 11: The velocity of the interface on the axis for $R / R_{\nu}=2.8810^{5}$. The oscillations correspond to the arrival of a train of capillary waves. For this large value of $R / R_{\nu}$ capillary waves are numerous and of short wavelength. The very large excursion in velocity may be due to the existence of a further bubble entrapment region, however the very small scales involved make numerical resolution difficult. 


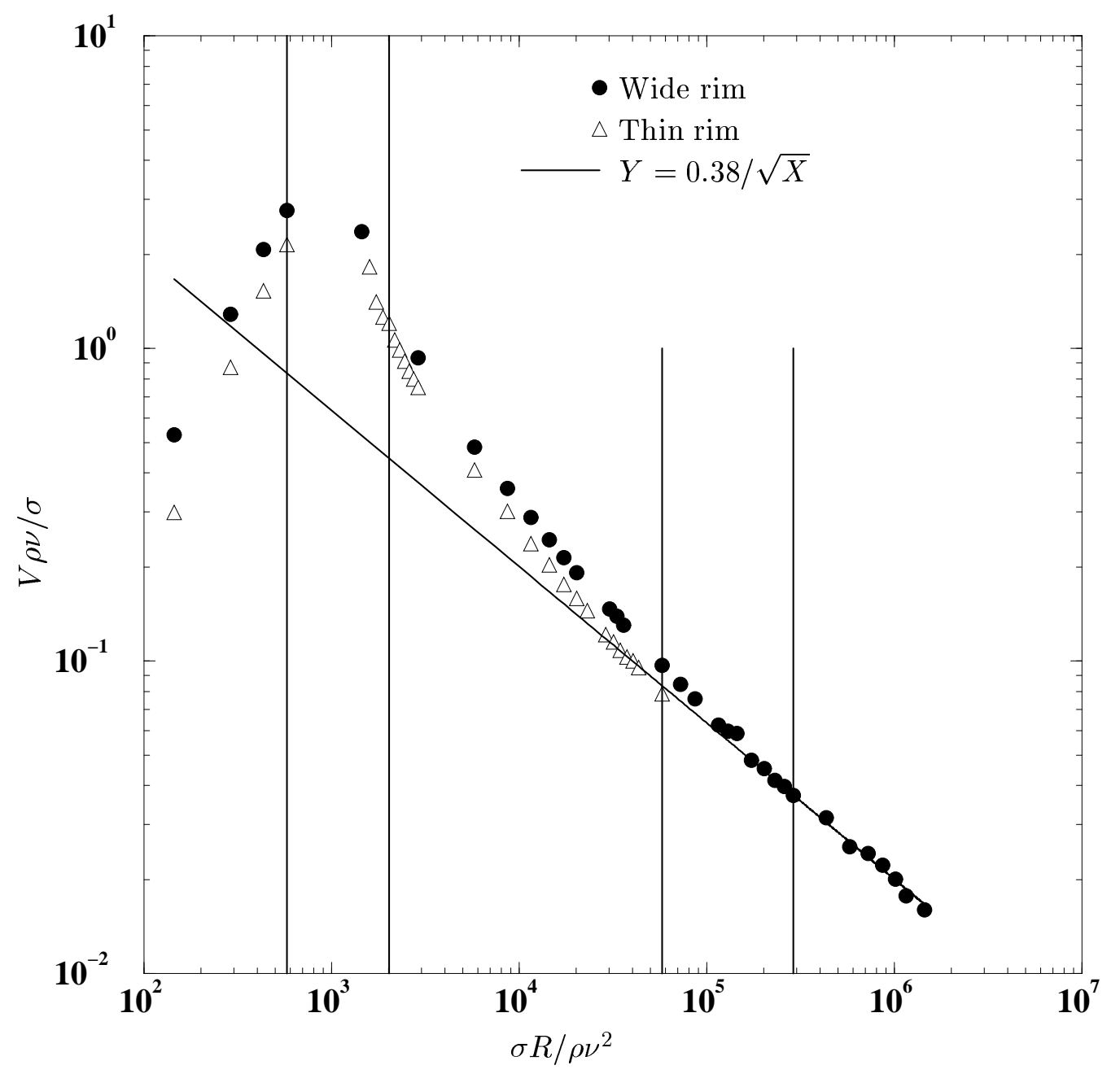

Figure 12: Non-dimensional jet velocity as a function of the non-dimensional bubble radius. The two regions between vertical straight lines correspond to the radii for which a bubble is trapped at the base of the jet . 
inviscid scaling. For small radii the velocity starts decreasing as $R$ decreases. For the smallest radii we have investigated the cavity relaxes to a flat surface shape without jet formation.

The regions where bubbles form at the base of the jet are indicated as vertical lines on Fig. 12. In the leftmost region, around $R / R_{\nu}=10^{3}$, for the reasons discussed above, there is a gap in data points. It is thus possible that much higher jet velocities may be reached in that region.

\subsection{Maximum pressure on the axis of symmetry}

We have computed the maximum pressure on the axis of symmetry when the jet reaches the mean water level. Fig. 13 shows this pressure and a fit in $\left(R / R_{\nu}\right)^{-1}$. Once more, the numerical result is in good agreement with the scaling law for radii between $2.10^{4}$ and $10^{6}$ times the viscous-capillary length. We also remark a small jitter about the straight line on the right hand side of the curve, perhaps as a result of the singular behavior in the bubble entrapment region. Note again that in the left hand side of the curve we could not reliably calculate pressure.

\subsection{Radius of the first ejected drop}

Experimental data obtained by Spiel et al. [6] tend to show that the radius of the first ejected drop is about one-tenth the radius of the initial bubble.

We have obtained this radius from the numerical simulations as follows. The computation stops when the jet thickness reaches the size of one computational cell. The jet rupture will occur soon thereafter. The volume enclosed by the free-surface between this point of minimum thickness and the tip of the jet is then a good approximation of the volume of the ejected droplet. The equivalent radius $R_{d}$ is defined as the radius of a spherical droplet with the same volume.

Fig. 14 shows $R_{d} / R$. For large $R / R_{\nu}$ we obtain a linear fit $R_{d} \approx 0.13 R$ which is consistent with the experimentally observed value of $R / 10$. This linear behavior is consistent with the viscosity-independent regime of Eqs. $(7,8)$ in which the only length scale is $R$. On the other hand, there is a large fraction of the data where this regime does not hold and the ejected drop radius is much smaller than $R / 10$. Notice again the gap in values around $R / R_{\nu}=10^{3}$. There the jet was too thin to be well-resolved numerically, and the actual droplet size may be much smaller. Varying the initial condition 


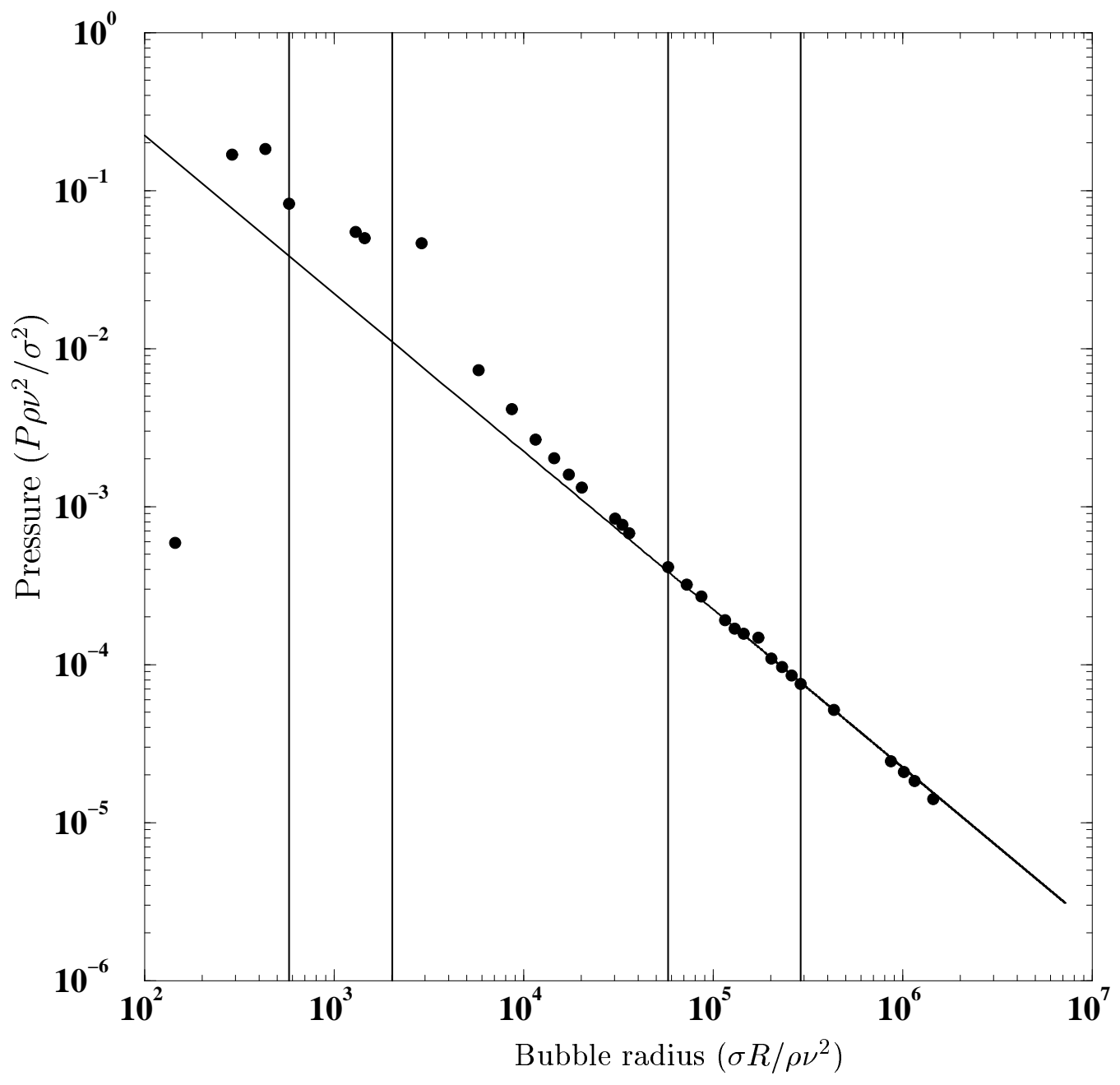

Figure 13: Maximum pressure on the axis of symmetry when the jet reaches the mean water level. As in the previous figure the bubble entrapment regions are marked. 


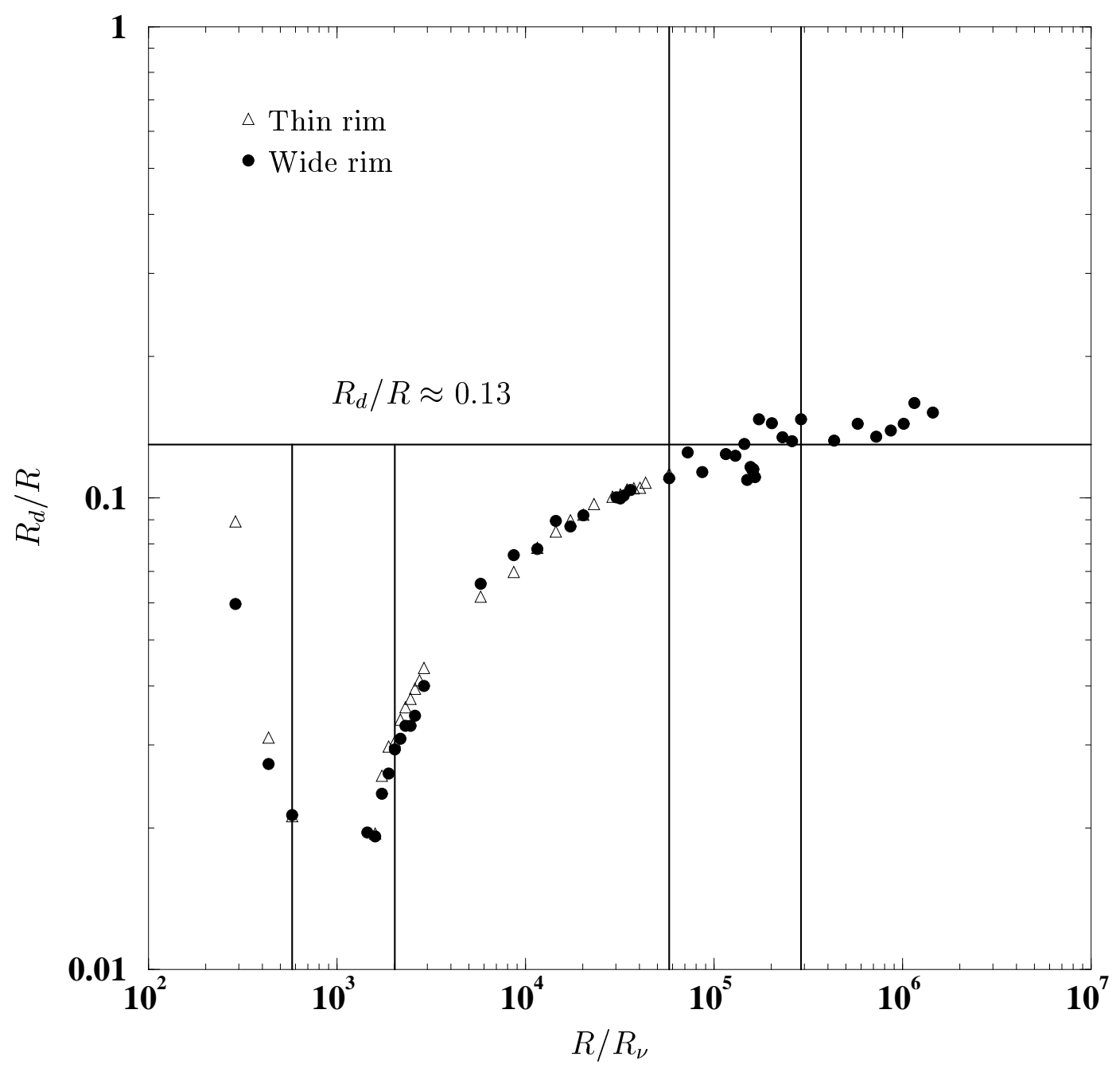

Figure 14: Ratio of the radius of the first ejected drop and the radius of the initial bubble as a function of $R / R_{\nu}$. 
has little effect, except at small radii where the thinner rim leads to a larger droplet.

\section{Singular jet formation by curvature rever- sal}

The formation of a thin, high-velocity jet in and around the first bubble entrapment region leads to suspect the existence of a singularity. The scaling (3) discussed above yields

$$
\begin{gathered}
h(u, t)=\left(t_{0}-t\right)^{2 / 3} f\left(u\left(t_{0}-t\right)^{-2 / 3}\right), \\
\Phi(u, z, t)=\left(t_{0}-t\right)^{1 / 3} \varphi\left(u\left(t_{0}-t\right)^{-2 / 3}, z\left(t_{0}-t\right)^{-2 / 3}\right),
\end{gathered}
$$

where $h$ is the surface elevation and $u=r \cos \theta$ the distance to the axis of symmetry. We rescaled the radial and vertical coordinates of the surface points by $\left(t_{0}-t\right)^{2 / 3}$ for $R / R_{\nu}=720$. We determined $t_{0}$ by fitting two of the rescaled profiles onto one another. The results are shown on Fig. 15.

All the profiles have been translated vertically in order for the point on the axis of symmetry to be at the same vertical coordinate. The rescaled profiles superimpose well at small values of the similarity variable $\eta$. The shape of the profiles closely resembles the experimental and numerical profiles in other types of flow $[11,14,9]$. However at a large distance from the singularity the cone angle is about $73^{\circ}$. This should be compared with the angle of the cavity seen in the McIntyre data shown on Fig. 3. There, on profile 6 we measure an angle of $68^{\circ}$, a small difference with our calculations. In contrast, the other physical processes discussed in the introduction yield relatively larger angles.

The finite viscosity should also introduce a discrepancy with the theoretical similarity solution. It seems however that its effects are small in that case.

In reference [9] it was shown that for Faraday waves there was a connection between bubble entrapment and singularities. In our case the picture seems different. The self-similar solution (10) is observed in the entire first bubble entrapment band. On the other hand, this solution is not seen in or around the second band of bubble entrapment, where we should in principle also have a singularity. However the shape of the interface is very different in that case (Fig. 16) and a superposition using the above rescaled variables 


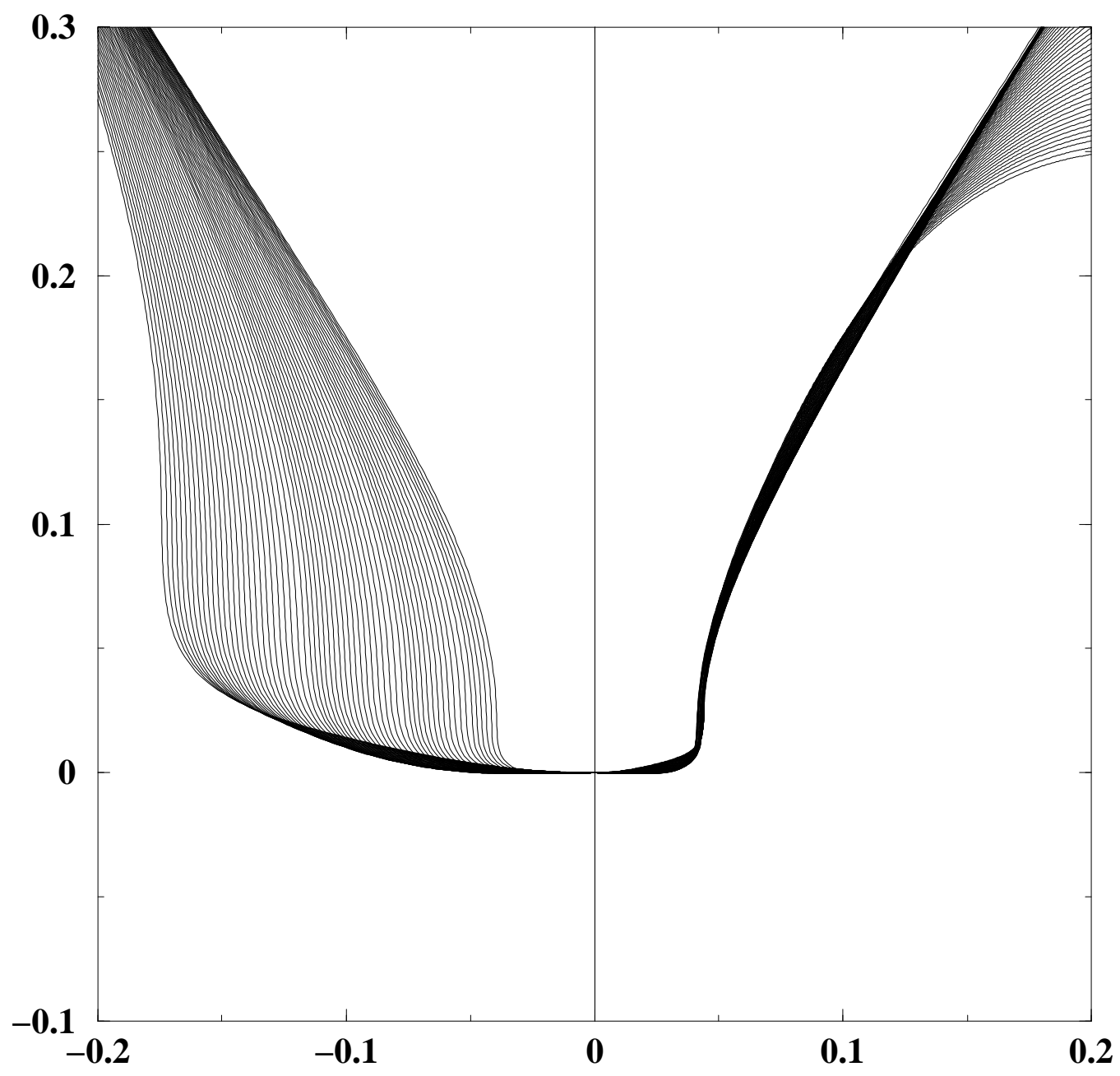

Figure 15: Comparison between successive profiles made non-dimensional using the scaling $\eta=x /\left(t_{0}-t\right)^{2 / 3}$ described in the text. Left: unrescaled profiles. Right: rescaled profiles. There the opening angle of the cavity is around $73^{\circ}$. Since the numerical method uses adaptive time-stepping, the profiles are not separated by equal time intervals. 


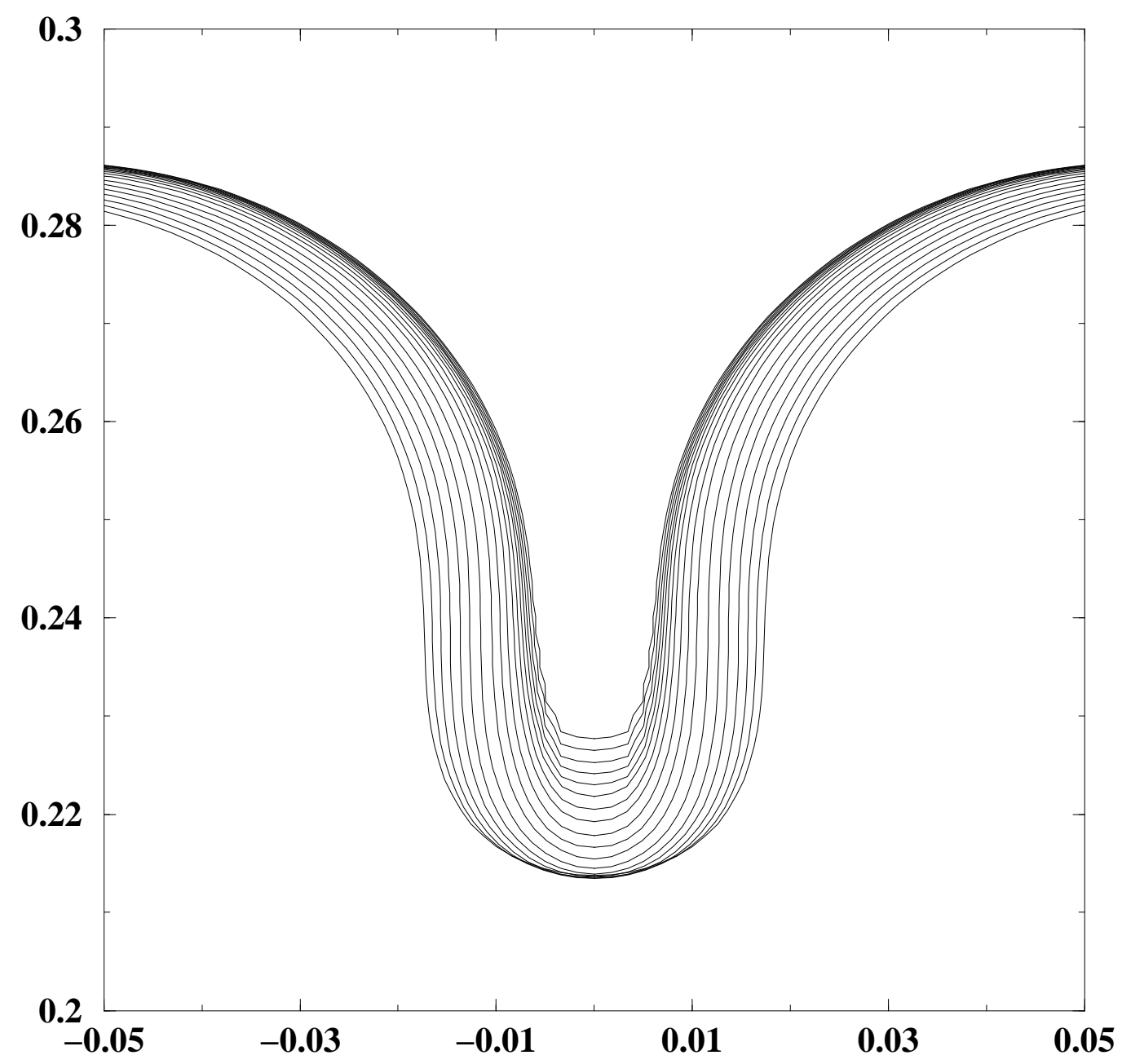

Figure 16: Shape of the interface on the edge of the second bubble formation region at $R=57600$. In that case a rescaling of the type shown on Fig 15 could not be found. As in the previous Figure profiles are not separated by uniform time intervals. 
could not be found. A possible explanation is that the conical cavity associated with the singularity is present here only on the large scale as seen on Fig. 10. The convergence of small-scale capillary waves is not able by itself to generate a self-similar conical flow. Thus the conical flow would be at least as important as bubble formation in producing the surface-tension driven self-similar scaling.

A tantalizing possibility is the existence of further bands of bubble entrapment and singularities to the right of the second band. While bubble entrapment is observed in some cases, details of the dynamics are not wellenough resolved. It is likely that bubble entrapment and cusp singularities are related to the amplitude of the converging capillary waves. As viscosity is reduced, ever more capillary waves are observed to converge on the axis. For very large values of $R / R_{\nu}$, waves having both short wavelength and small amplitude are formed. 


\section{Conclusions}

We have presented a numerical study of the bursting process of bubbles at a free surface. The scheme used was based on an accurate description of the free surface with the help of a markers chain. This method has shown good capabilities to resolve small capillary waves. The large scale features of the dynamics, the pressure and final droplet radius may be predicted with accuracy, except near the first bubble entrapment region near $R / R_{\nu}=10^{3}$. The predictions are quantitatively in agreement with experiment: the angle of opening of the cavity is similar to the angle observed in the experiments of MacIntyre and the size of the droplet at the tip of the jet is close to the experimentally reported size.

The measurements of jet velocity near $R / R_{\nu}=10^{3}$ show a surprisingly large velocity. The interface shape scales with a characteristic length $r \sim$ $\left|t-t_{0}\right|^{2 / 3}$ predicted by the balance of surface tension and inertia. The shape of the interface resembles shapes found in other jet-forming flows and cusp singularities, but has quantitative differences such as the opening angle of the conical cavity.

The connection of this scaling with bubble entrapment is less clear. We found the scaling in a wide region. The occurrence of self similar flow and an approximate singularity is not connected to the exact boundary of a bubble entrapment band. We also found bubble entrapment transitions which were not associated to the $\left|t-t_{0}\right|^{2 / 3}$ scaling. Finally the angle of the conical cavity agrees with the experimental data for bursting bubbles, but not with the angles seen or predicted in other flows. This indicates that other types of singularities, corresponding to different topologies or initial conditions, may be observed. Further work should explore in detail the nature of these singularities using for instance mesh refinement.

Also of interest would be a study of the influence of the initial shape of the bubble. We have shown that a factor of two change in the rim thickness had no qualitative effect, and very little quantitative effect on the collapse process. However other changes in the initial condition may cause a change in the position of the various singularities. In other words, for a given radius, it would be possible to reach a singularity by changing the shape of the bubble. 


\section{References}

[1] M. Coantic. Mass transfert across the ocean-air interface : small scale hydrodynamic and aerodynamic mechanisms. PhysicoChemical Hydrodynamics, 1:249-279, 1980.

[2] J.M. Boulton-Stone and J.R. Blake. Gas bubbles bursting at a free surface. J. Fluid Mech., 254:437-466, 1993.

[3] J. M. Boulton-Stone. The effect of surfactants on bursting gas bubbles. J. Fluid Mech., 302:231-257, 1995.

[4] M. Rudman. A volume-tracking method for incompressible multi-fluid flows with large density variations. Int. J. Numer. Meth. Fluids, 28:357378, 1998.

[5] D. C. Blanchard. The size and height to which drops are ejected from bursting bubbles in seawater. J. Geophys. Res., 94(C8):10999-11002, 1989.

[6] D. E. Spiel. On the births of jet drops from bubbles bursting on water surfaces. J. Geophys. Res., 100(C3):4995-5006, 1995.

[7] F. MacIntyre. Flow patterns in breaking bubbles. J. Geophys. Res., 77(27):5211-5228, 1972.

[8] M. S. Longuet-Higgins. Bubbles, breaking waves and hyperbolic jets at a free surface. J. Fluid Mech., 127:103-121, 1983.

[9] B. W. Zeff, B. Kleber, J. Fineberg, and D. P. Lathrop. Singularity dynamics in curvature collapse and jet eruption on a fluid surface. Letters to Nature, 403:401-404, 2000.

[10] M. S. Longuet-Higgins and H. Oguz. Critical microjets in collapsing cavities. J. Fluid Mech., 290:183-201, 1995.

[11] H. N. Oguz and A. Prosperetti. Bubble entrainment by the impact of drops on liquid surfaces. J. Fluid Mech., 203:143-179, 1990.

[12] M. S. Longuet-Higgins. An analytic model of sound production by raindrops. J. Fluid Mech., 214:395-410, 1990. 
[13] M. Rein. The transitional regime between coalescing and splashing drops. Journal of Fluid Mechanics, 306:145-65, 1996.

[14] D. Morton, Liow Jong-Leng, and M. Rudman. An investigation of the flow regimes resulting from splashing drops. Physics of Fluids, 12(4):747-63, 2000.

[15] J.B. Keller and M.J. Miksis. Surface tension driven flows. SIAM Journal on Applied Mathematics, 43(2):268-77, 1983.

[16] D. C. Blanchard and L. D. Syzdek. Film drop production as a function of bubble size. J. Geophys. Res., 93(C4):3649-3654, 1988.

[17] S. Popinet and S. Zaleski. A front tracking algorithm for the accurate representation of surface tension. Int. J. Numer. Meth. Fluids, 30:775793, 1999.

[18] S. Popinet and S. Zaleski. Bubble collapse near a solid boundary : a numerical study of the influence of viscosity. Submitted to JFM. 